\title{
El papel de los organismos internacionales en el diseño de políticas públicas de desarrollo*
}

\author{
The role of international organizations in the design \\ of public development policies \\ O papel dos organismos internacionais na elaboração \\ de políticas públicas de desenvolvimento
}

\section{Edgar Ricardo Rodríguez Hernández ${ }^{1}$}

Recibido: 18 de septiembre de 2018

Aceptado: 6 de mayo de 2019

Publicado: 21 de julio de 2019

Cómo citar este artículo: Rodríguez Hernández, E. R. (2019). El papel de los organismos internacionales en el diseño de políticas públicas de desarrollo. Cooperativismo \& Desarrollo, 27(2), 1-29. doi: https://doi.org/10.16925/2382-4220.2019.02.05

* $\quad$ Artículo de de investigación. https://doi.org/10.16925/2382-4220.2019.02.05

1 Maestro en Negocios y Estudios Económicos. Estudiante del Doctorado en Políticas Públicas y Desarrollo en el Centro Universitario de Ciencias Económico Administrativas de la Universidad de Guadalajara. Profesor en la Licenciatura en Administración de Negocios y Contaduría Pública del Centro Universitario de Tonalá, Jalisco, México. Correo electrónico: edgar.rhernandez@aca-demicos.udg.mx ORCID: https://orcid.org/0000-0001-8915-218X 


\title{
Resumen
}

Este artículo tiene como objetivo examinar teórica y empíricamente el papel de los organismos internacionales en el diseño de políticas públicas de desarrollo bajo un contexto de relaciones de poder asimétricas entre actores estatales y no estatales. En este sentido, se identifica que los factores determinantes que influyen en el papel de los organismos internacionales para formar coaliciones con actores estatales y no estatales en el diseño de políticas de desarrollo son la estructura de poder y la confianza. Se presenta una serie de estrategias con las que un organismo internacional, independientemente de la especialización, podría mejorar tales aspectos, entre las que destacan: cambio en el discurso ideológico de desarrollo, disminución de la autonomía de los grupos de poder o élite de la política pública, promoción de la transparencia institucional, la rendición de cuentas y seguimiento posapoyo de desarrollo.

Palabras clave: organismo internacional, políticas de desarrollo, actores estatales, actores no estatales

\begin{abstract}
his article aims to theoretically and empirically examine the role of international organizations in the design of public development policies under a context of asymmetric power relations between state and non-state actors. In this sense, it has been identified that the determining factors that influence the role of international organizations in forming coalitions with state and non-state actors in the design of development policies are the power structure and trust. A series of strategies are presented with which an international organization, regardless of specialization, could improve such aspects, among which are: change in the ideological discourse of development, decrease in the autonomy of groups with power or the elite within public politics, promotion of institutional transparency, accountability and post-development support monitoring.
\end{abstract}

Keywords: international body, development policies, state actors, non-state actors.

\section{Resumo}

Este artigo tem como objetivo examinar teórica e empiricamente o papel dos organismos internacionais na elaboração de políticas públicas de desenvolvimento sob um contexto de relações de poder assimétricas entre atores estatais e não estatais. Nesse sentido, identifica-se que os fatores determinantes que influenciam o papel dos organismos internacionais para formar coalizões com atores estatais e não estatais na elaboração de políticas de desenvolvimento são a estrutura de poder e a confiança. São apresentadas estratégias com as quais um organismo internacional, independentemente da especialidade, poderia melhorar esses aspectos, entre as quais se destacam: mudança no discurso ideológico de desenvolvimento, diminuição da autonomia dos grupos de poder ou elite da política pública, promoção da transparência institucional, prestação de contas e seguimento pós-apoio de desenvolvimento.

Palavras-chave: atores estatais, atores não estatais, organismo internacional, políticas de desenvolvimento. 


\section{Introducción}

A partir de la segunda mitad del siglo XIX las interacciones económicas, político-institucionales, científico-técnicas, culturales, militares y sociales, entre los actores internacionales ${ }^{2}$, han experimentado importantes transformaciones como consecuencia de los regímenes internacionales que se han visto reformados ante la nueva configuración de poder, así como de la creciente interdependencia y la globalización cuyas dinámicas han marcado las pautas del comportamiento de los actores tanto públicos como privados para diseñar políticas que resuelvan problemas contemporáneos de desarrollo ubicados en la agenda pública internacional.

Cabe destacar que el diseño de políticas de desarrollo implica planificar y administrar los recursos disponibles de un país, una región o localidad de manera estratégica, reconocer los actores clave en el desarrollo económico y establecer mediante el consenso las prioridades sobre objetivos de desarrollo y estrategias específicas para dar solución real a los problemas que limitan el bienestar de la sociedad en su conjunto (Robinson, 2014; Fernández y Schejtman, 2012).

Al consultar los objetivos y las metas de la agenda internacional 2030 para el desarrollo sostenible de las Naciones Unidas se considera que durante los próximos doce años se requerirán diseñar o, en su caso, mejorar las políticas de desarrollo. Ello, en las siguientes esferas de importancia crítica para la humanidad y el planeta, a saber: persistencia de la pobreza, desigualdad de género, cambio climático, disponibilidad y calidad del agua, eficiencia energética, empleabilidad tanto en zonas urbanas como rurales, degradación de las tierras y pérdida de la diversidad biológica, terrorismo global, virus y pandemias, resurgimiento del etnonacionalismo, peligros de la proliferación de armas nucleares, crecimiento continuo de la migración internacional, crisis financieras y crimen organizado.

Esimportanteresaltarqueningunodeestosproblemasglobalescontemporáneos puede ser resuelto por Estados que actuén solos. Todos requieren coalición coordinada entre los Estados y con actores no estatales (Karns, et al. 2015); todos están tan interrelacionados que las instituciones y las políticas tradicionales ya no son capaces de hacer frente a ellos por lo que se requiere promover nuevas iniciativas, reglas de negociación y acciones políticas que consideren las situaciones conflictivas, dinámicas y complejas en la interrelación entre actores estatales y no estatales (Ocampo, 2013; Meadows, et al. 1972). Algunos exigen mecanismos internacionales de supervisión

2 Se entiende por actores internacionales aquellos actores racionales públicos y privados que se interrelacionan en la arena política internacional y cuyo comportamiento incide en la vida de la sociedad internacional. 
para asegurar el cumplimiento de normas o patrones de comportamiento por parte de los actores internacionales (Querejazu, 2013); muchos requieren nuevos tipos de alianzas, algunas entre organizaciones internacionales existentes y algunas otras entre asociaciones público-privadas (Karns, et al. 2015).

En resumen, en la actualidad existe una gran variedad de problemas transfronterizos que para poderlos paliar y solucionar se requiere diseñar alternativas de solución globales, pues los problemas que impactan a la mayoría de las economías se agudizan y se trasmiten de manera rápida y sistémica. En este sentido, Giraldo (2015) y Karns, et al. (2015) coinciden en determinar que para hacer frente a la complejidad de estos problemas globales de desarrollo es necesario llevar a cabo un proceso de integración de agendas y actividades para el desarrollo sostenible mediante un sistema de gobernanza global que fomente, impulse y coordine la coalición entre actores del sector público y privado para alcanzar metas colectivas.

Para analizar esta propuesta, es importante esclarecer que gobierno global y gobernanza global no es lo mismo. Es común que se utilicen estos conceptos como si fueran sinónimos cuando en realidad no lo son. Al respecto, Giraldo (2015) señala que tanto el gobierno como la gobernanza global se refieren a sistemas de gobierno y acciones orientadas a cumplir objetivos; pero el gobierno se estructura a partir de una autoridad formal con poderes políticos, mientras que la gobernanza global:

La gobernanza tiene en cuenta la presencia de instituciones gubernamentalesy también de los mecanismos informales y no gubernamentales para la toma de decisiones. No pretende sustituir los gobiernos nacionales por un gobierno supranacional, sino que se concentra en generar un nuevo mecanismo para definir reglas de juego y para la toma de decisiones, que garantice mayores niveles de democracia, donde el ciudadano no se limite a hacer escuchar su voz a través de la democracia representativa, sino que se apoye en la democracia deliberativa, para que en vez de perder poder y protagonismo, el ciudadano sea protagonista en el escenario mundial. (Giraldo, 2015, p. 11).

Al contrastar ambos sistemas de gobierno se discurre; por un lado, que es inviable establecer un gobierno global o un sistema dominado por un Estado ya que se estaría generando un imperio mundial; y, por el otro, se observa que, al menos teóricamente, en la gobernanza global no existe una estructura de poder autoritaria centralizada o jerárquica de arriba hacia abajo, más bien se pretende impulsar el sistema democrático deliberativo entre los actores internacionales. No obstante, se considera que este 
sistema de gobierno aunque podría ser considerado como "el ideal" para impulsar la democratización no es consistente con la realidad ya que es común que se susciten relaciones de poder asimétricas y conflicto de intereses en el proceso de toma de decisiones entre los actores internacionales. En consecuencia, se establecen actores ganadores y perdedores y, especialmente, se desvaloriza la cooperación entre actores estatales y no estatales en el diseño de políticas de desarrollo por lo que estas tienden a ser centralizadas y diseñadas por un grupo de poder.

De esta manera, se arguye que la discusión no es sobre si un sistema de gobernanza global es viable o no, sino más bien, ante las relaciones de poder asimétricas, conflicto de intereses, la globalización y la cambiante situación internacional. Asi, las preguntas son: ¿cómo hacer que este sistema de gobernanza global sea efectivo e incluyente en el diseño de políticas de desarrollo?, y ¿cuál (es) será (n) el (los) actor (es) que dirigirá (n) el rol funcional de la gobernanza para la sociedad global? Al respecto, Ocampo (2015; 2013) analiza cuáles serían las mejores estructuras de gobernanza global tomando en cuenta ocho criterios: 1) El reconocimiento del principio de subsidiariedad. 2) Una red densa de instituciones mundiales, regionales y nacionales. 3) Superar la tensión entre la inclusividad y la legitimidad asociada a ella. 4) Una participación equitativa de los países en vías de desarrollo en la toma de decisiones y la fijación de normas. 5) Idear instrumentos eficaces de monitoreo, rendición de cuentas y cumplimiento de los compromisos internacionales. 7) Provisión equitativa de bienes públicos globales. 8) Diseño e implementación de política de desarrollo integral eficiente y efectiva.

Con base en lo anterior y al examinar las aportaciones de Enríquez (2015) y Babb y Chorev (2016) es posible argumentar que los Organismos Internacionales (OIs) son los arreglos institucionales que más se aproximan a cumplir con los criterios señalados por Ocampo (2015; 2013). Dado su estructura, objetivos y funciones los Ols cumplen con la mínima institucionalidad que regula el sistema mundial, es una de las entidades que mayor poder político-institucional, científico-técnico, epistemológico y financiero despliegan en manejar la interdependencia, superan gradualmente las asimetrías de información y poder que caracterizan al sistema económico mundial así como en integran, cabildean y coordinan acciones colectivas que mejoran las condiciones socioeconómicas, políticas y ambientales entre y al interior de los países a través de la cooperación multilateral al desarrollo.

Con base en Restrepo (2013) y Álvarez (2012) se discurre que, en el área de la cooperación multilateral al desarrollo; ni los Estados por sí solos ni los Ols pueden hacer frente a los problemas, riesgos y retos de la sociedad internacional contemporánea. Se requiere la participación de nuevos actores no estatales, los cuales debido a 
su protagonismo y dinamismo han ocupado una parte cada vez más amplia e importante en la cooperación internacional y especialmente en el proceso de negociación y consenso en el diseño de políticas de desarrollo. Concretamente Álvarez (2012) aporta una clasificación de las fuentes, los tipos de cooperación y los actores que intervienen en la cooperación internacional (ver tabla 1).

Tabla 1. Fuentes de la cooperación internacional

\begin{tabular}{|c|c|c|}
\hline Fuentes & Tipos de cooperación & Actores \\
\hline $\begin{array}{l}\text { Cooperación } \\
\text { gubernamental }\end{array}$ & $\begin{array}{ll}\text { - } & \text { Bilaterales } \\
\text { - } & \text { Multilaterales } \\
\text { - } & \text { Descentralizados }\end{array}$ & - Gobiernos (nacionales y locales) \\
\hline $\begin{array}{l}\text { Cooperación no } \\
\text { gubernamental }\end{array}$ & $\begin{array}{l}\text { - Privados } \\
\text { - Organizaciones de la sociedad civil } \\
\text { - Universidades }\end{array}$ & $\begin{array}{l}\text { - } \\
\text { - } \text { Organizaciones No Gubernamentales } \\
\text { - } \quad \text { Sociedad civil } \\
\text { - Universidades }\end{array}$ \\
\hline Fondos mixtos & - Gubernamentales y no gubernamentales & - Mixtos \\
\hline
\end{tabular}

Fuente: Álvarez (2012, p. 299)

En virtud de lo señalado, y con base en la tabla 1, se discurre que los Ols para cumplir con los objetivos de la gobernanza global y formar coaliciones en el diseño de la política de desarrollo dependen del comportamiento y estructura del poder que son resultados de las ideologías, preferencias, motivaciones e intereses del Estado y de los nuevos actores no estatales (Sanahuja, 2008). Se considera que la heterogeneidad de estos elementos coadyuva en múltiples ocasiones a que existan relaciones de poder asimétricas y conflictos de intereses que dificultan y desincentivan a los actores internacionales a formar coaliciones en el diseño de políticas de desarrollo.

Asimismo, otras de las principales causas por las que los actores internacionales deciden no colaborar en la formación de dichas coaliciones son: las contradicciones teórico-prácticas de los elementos clave del multilateralismo -acuerdo, incluyente, igualitario, control y supervisión (Caamaño, 2014)-; mecanismos inadecuados en la gobernanza global para hacer cumplir el derecho internacional (Stiglitz, 2013); la crisis de confianza y débiles mecanismos institucionales para la rendición de cuentas (Bárcena, 2015); y "no existen normas específicas para tratar la discusión sobre le legalidad de actos ultra vires, ni mecanismos de impugnación ni controles de legalidad de los actos de los Ols" (Querejazu, 2013, p. 2).

Con base en lo anterior es posible argumentar que, en el contexto internacional, la calidad de los resultados del proceso de formulación de política de desarrollo depende 
tanto del funcionamiento de los Ols como de las coaliciones y reglas institucionales que determinen los roles de los actores estatales y no estatales matizando que los principales retos que afrontan los Ols para formar dichas coaliciones recaen en aspectos como la estructura del poder, la confianza y capacidad de los funcionarios de los Ols para negociar y hacer cumplir acuerdos políticos en tiempo y forma. No obstante, es importante señalar que dichos estudios no esclarecen algunas cuestiones como: ¿cuáles son los intereses, discursos, estrategias, así como las principales funciones, limitaciones y los alcances de los Ols en torno al diseño de la política de desarrollo?, ¿cómo se forjan las preferencias en los Estados para participar o salir de un Ol? En suma, dichos estudios no dan propuestas o estrategias sobre cómo los Ols podrían mejorar su papel en el diseño de políticas desarrollo.

El interés de este trabajo de investigación al tratar de responder tales cuestionamientos es ir más allá de estudiar a los Ols como sujetos de derecho o como actores subordinados a los países hegemónicos que los conforman. Lo relevante es concebir a los Ols como el arreglo institucional que puede fungir como el actor y coordinador de la gobernanza global, que conforman junto con otros actores estatales y no estatales redes de poder que inciden tanto en el diseño de políticas de desarrollo como en las condiciones sociales, económicas, políticas y ambientales entre y al interior de los países. De esta manera, el objetivo general de este trabajo de investigación es examinar tanto teórica como empíricamente el papel de los organismos internacionales en el diseño de políticas públicas de desarrollo bajo un contexto de relaciones de poder asimétricas entre actores estatales y no estatales.

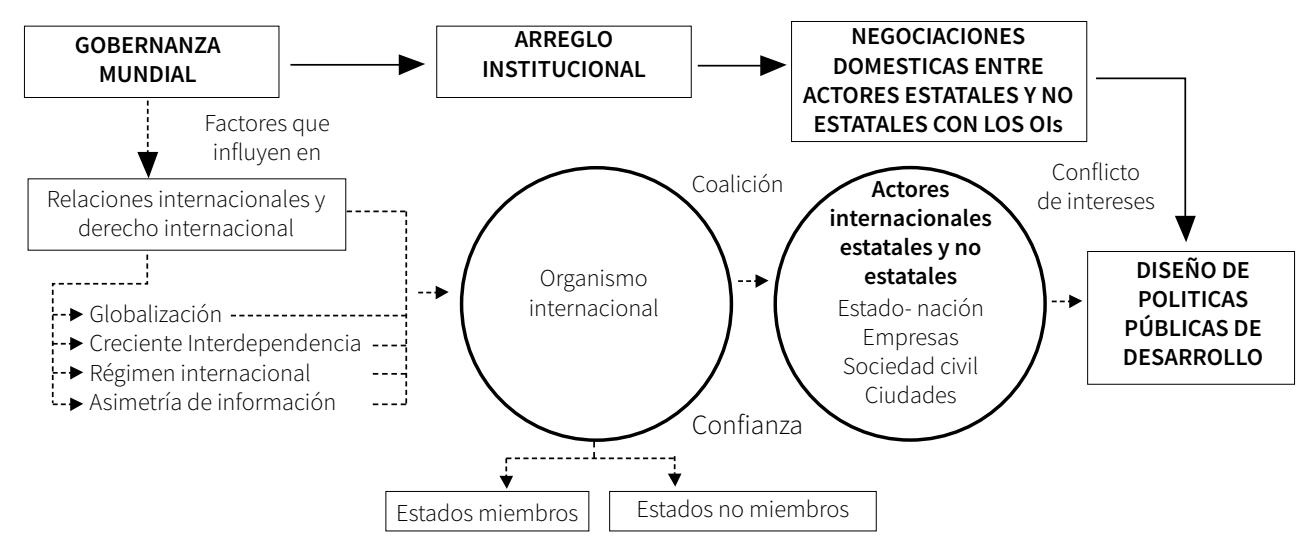

Figura 1. Dinámica y transformaciones de los mercados internos, la economía mundial y la política internacional.

Fuente: elaboración propia 
Para responder los cuestionamientos planteados y cumplir con el objetivo esbozado, en este trabajo de investigación se propone seguir la trayectoria de la figura 1 que denota los factores que inciden en el diseño de las políticas de desarrollo en el contexto internacional. En este sentido, este artículo se divide en tres apartados, más la presente introducción. En el primer apartado se presenta el contexto bajo el que se sitúa el objeto de estudio, es decir, se describe el cambio de paradigma de régimen internacional y se analiza la globalización como marco de las relaciones internacionales. En el segundo apartado se presenta una revisión de la literatura que discute tanto teórica como empíricamente por qué los Estados deciden incursionar o salirse de un Ols, así como los intereses, los discursos y las estrategias de los Ols para formar coaliciones con actores estatales -Estado-nación- y no estatales -empresas transnacionales y sociedad civil- en el diseño de políticas de desarrollo; finalmente, en el tercer apartado se ofrecen las conclusiones y, además, con base en la previa revisión de la literatura, se presentan algunas estrategias que podrían implementar los Ols para mejorar su papel en el diseño de políticas desarrollo.

\section{La globalización como marco de las relaciones internacionales}

En la literatura sobre regímenes internacionales se encuentran diversos estudios que señalan que el Estado perdió de manera significativa su protagonismo para gobernar. Esto debido, en gran medida, a su incapacidad institucional para responder con efectividad a los cambios que ocurrían en su entorno como la globalización, la creciente interdependencia, el poder de los mercados internacionales, los problemas globales entre otros. Por lo anterior era necesario proporcionar alternativas que permitieran dar una dirección efectiva a la economía y mejorar las condiciones de vida de la sociedad internacional a través de acciones colectivas; como establecer una red de gobernanza multinivel (Stiglitz, 2006).

Según Stiglitz (2006) dicha situación ha provocado que se presente un cambio de paradigma respecto al régimen internacional contemporáneo. Es decir, se ha transformado el clásico gobierno estatocéntrico del modelo realista a una red de gobernanza multinivel. Esto bajo la justificación que la red de gobernanza multinivel es un sistema más eficaz de cooperación internacional que permite, entre otros aspectos, "lograr que algunos intereses, que de otra manera quedarían excluidos, tengan una mayor oportunidad de participar en el sector público" (Guy, 2007, p. 42). Se considera que el debate entre quienes ven al Estado como la institución clave o protagonista en la política 
internacional y aquellos que ven la necesidad de una regulación política que vaya más allá del Estado considerando a las instituciones internacionales no jerárquicas como deseables es un buen punto para iniciar la discusión sobre el papel de los Ols, tanto en las relaciones internacionales como en la formulación de políticas de desarrollo.

En este sentido, en Salomón (2002) y Baldwin (1993) se identificaron las principales líneas de discusión entre el sistema internacional realista y el globalismo que después se convertiría en el debate entre neorrealismo y neoliberalismo (ver tabla 2).

Tabla 2. Debates entre neorrealistas y neoliberales en la interpretación de la realidad internacional.

\begin{tabular}{|c|c|c|}
\hline \multicolumn{3}{|c|}{ Regímenes internacionales } \\
\hline Divergencias & Neorrealismo & Neoliberalismo \\
\hline Precursor & Kenneth Waltz en 1979 & Robert Owen Keohane en 1983 \\
\hline $\begin{array}{l}\text { Principal (es) actor } \\
\text { (es) internacional } \\
\text { (es) en la política }\end{array}$ & $\begin{array}{l}\text { Los Estados -unidades racionales y } \\
\text { autónomas- son los principales actores de la } \\
\text { política internacional debido a dos atributos } \\
\text { que lo diferencian de cualquier otra entidad } \\
\text { internacional: la territorialidad y la soberanía. }\end{array}$ & $\begin{array}{l}\text { Los Estados han venido perdiendo } \\
\text { protagonismo, mientras que los actores } \\
\text { no estatales han aumentado su poder, } \\
\text { alcance e influencia en la formulación de } \\
\text { política. }\end{array}$ \\
\hline $\begin{array}{l}\text { Cooperación } \\
\text { internacional }\end{array}$ & \multicolumn{2}{|c|}{$\begin{array}{l}\text { La cooperación internacional es más difícil de lograrse, más difícil de mantenerse y } \\
\text { más dependiente de las relaciones de poder de los estados que lo que afirman los } \\
\text { neoliberales. En el neorrealismo el poder es la principal categoría analítica de la teoría, y } \\
\text { la anarquía es la característica definitoria del sistema internacional. }\end{array}$} \\
\hline $\begin{array}{c}\text { Beneficios } \\
\text { absolutos/relativos }\end{array}$ & $\begin{array}{l}\text { Los Estados, al iniciar la cooperación con otros, } \\
\text { buscan ante todo mejorar su posición relativa } \\
\text { frente a los demás. De ahí que teman que otros } \\
\text { estados puedan obtener ganancias mayores } \\
\text { que ellos en la cooperación (superarlos en } \\
\text { ganancias relativas). Este temor es lo que } \\
\text { impediría la cooperación a largo plazo. }\end{array}$ & $\begin{array}{l}\text { Consideran que en general prevalecerá } \\
\text { el deseo de obtener beneficios absolutos } \\
\text { (deseo que llevará a intentar maximizar } \\
\text { el nivel total de los beneficios de quienes } \\
\text { cooperan) }\end{array}$ \\
\hline $\begin{array}{l}\text { Las prioridades de } \\
\text { las metas estatales }\end{array}$ & \multicolumn{2}{|c|}{$\begin{array}{l}\text { Ambos enfoques entienden que tanto la seguridad como el bienestar económico son } \\
\text { metas importantes, pero suelen diferir en cuanto a cuál de ellas es prioritaria para los } \\
\text { estados. Los neorrealistas ponen el énfasis en la seguridad mientras que los neoliberales } \\
\text { temas económicos. }\end{array}$} \\
\hline Coordinación & Burocrática dirigida & Negociada por consenso \\
\hline $\begin{array}{l}\text { Resolución de } \\
\text { conflictos }\end{array}$ & Poderes equilibradores & Mediación multilateral, consenso \\
\hline $\begin{array}{c}\text { Relaciones } \\
\text { público-privado }\end{array}$ & Primacía política & Conjunto público-privado \\
\hline $\begin{array}{l}\text { El papel de los } \\
\text { regímenes y las } \\
\text { instituciones }\end{array}$ & $\begin{array}{l}\text { Los neorrealistas consideran exagerado } \\
\text { el papel que atribuyen los neoliberales a } \\
\text { regímenes e instituciones }\end{array}$ & $\begin{array}{l}\text { Los regímenes y las instituciones } \\
\text { internacionales mitigan los efectos limitantes } \\
\text { que tiene la anarquía sobre la cooperación. }\end{array}$ \\
\hline $\begin{array}{l}\text { Moralidad y } \\
\text { la legitimidad } \\
\text { democrática de la } \\
\text { política de poder }\end{array}$ & $\begin{array}{l}\text { Hay una tendencia a ver la tradición realista } \\
\text { como amoral. Debido a las disparidades de } \\
\text { poder entre los estados, sólo los intereses } \\
\text { de quienes viven en estados poderosos } \\
\text { determinan los resultados internacionales. }\end{array}$ & $\begin{array}{l}\text { La afirmación moral hecha en este contexto } \\
\text { por los defensores de una perspectiva } \\
\text { internacionalista es que un sistema estatal } \\
\text { multilateralista es más democrático que un } \\
\text { sistema estatal competitivo. }\end{array}$ \\
\hline
\end{tabular}

Fuente: elaboración propia con base en Salomón (2002); Baldwin (1993) 
Con base en la tabla 2 es posible argumentar que el cambio de paradigma reconoce que la corriente neoliberal cierne sus principios teóricos en la globalización, por lo que es posible puntualizar que la apertura de las economías dio paso a una mayor $-\mathrm{y}$ muchas veces conflictiva- interacción entre los diferentes actores internacionales. En materia política se observa que la política pública se desestatalizó y aparecieron actores no estatales con mayor poder, alcances e influencias en el diseño de la política pública. Por ello, el poder, los intereses, la capacidad de negociación y racionalidad de los actores internacionales se matizaron como variables determinantes en la cooperación internacional y en la obtención de beneficios absolutos/relativos. Por otra parte, se discurre que el cambio de paradigma provocó que el centro de interés fuera el estudio y análisis de la capacidad de los Ols para incentivar, cabildear y coordinar las negociaciones entre actores estatales y no estatales en la escena de la cooperación democrática internacional y formulación de políticas públicas. Finalmente, al analizar los criterios anteriores se destaca que el fenómeno de la globalización ha sido abordado bajo distintas perspectivas, algunos con opinión positiva (Allard, 2007) y otros con opiniones desalentadoras (Guy, 2007). Sin entrar en esta polémica, en este trabajo de investigación la atención se centra en discutir ¿cómo influye la globalización -si es que lo hace- en la cooperación democrática internacional?

En relación con este cuestionamiento se pueden encontrar opiniones contrapuestas. Es decir, por un lado se encuentran aquellos estudios que señalan que la cooperación democrática internacional adquiere más importancia con la globalización pues existe una tendencia creciente a actuar multilateralmente en vez de unilateralmente (Stiglitz 2013; Barkin, 2006); de otro lado, están aquellos que consideran que la cooperación democrática en un contexto globalizado es difícil de conceptualizar e incluso de sostener dado el déficit democrático que plantea la globalización (Guy, 2007; Albi, 2005). Particularmente, Albi (2005, p. 16) señala que la gobernación global a través de la cooperación internacional adquiere legitimidad democrática, sólo si el equilibrio de poderes impide la hegemonía de algún (os) Estado (s) que la constituyen sobre otros. Por otra parte, Guy (2007, p. 42) externa que "[...] el sistema internacional, por naturaleza, no es democrático, y las instituciones que tienen poder para tomar decisiones tienden a involucrar a actores nacionales más que a ciudadanos o intereses determinados".

Bajo esta última perspectiva es posible señalar que la dinámica de la globalización en cuanto a la cooperación internacional al desarrollo está determinada por la incipiente, desigual y conflictiva participación en la toma de decisiones entre los actores participantes. Existen actores ganadores y perdedores debido, en gran medida, a la distribución y estructura del poder. Por lo tanto, se considera que el poder 
es una de las variables más significativas que influye en la toma de decisiones de los actores internacionales en cuanto a cooperación en la formulación de políticas de desarrollo. En este sentido, existen estudios que definen y abordan de diferentes formas al poder, sin embargo, este trabajo adopta la definición de Del Arenal (1983, p. 509) ya que lo define -el poder- considerando el contexto internacional:

El poder es un medio a través del cual los actores se relacionan unos con otros tratando de influenciar su conducta. Implica concretamente la posesión de una serie de recursos — naturales, económicos, políticos, tecnológicos, intelectuales-que fundamentan en mayor o menor medida la habilidad o capacidad de un actor para actuar en las relaciones internacionales.

Con base en Del Arenal (1983) y Keohane y Nye (1977) se deduce que la clave del poder en un mundo multilateralista ya no proviene principalmente de la amenaza de la fuerza militar sino de la distribución y estructura del poder, la dependencia interestatal y la movilización y uso de los recursos tangibles e intangibles a disposición del actor que apoyan sus actos e intereses. Por otro lado, siguiendo a Dahl (1957), se discurre que el poder dado el nivel y capacidad de negociación e influencia en las preferencias y comportamiento de los actores provoca que se susciten dos escenarios en las relaciones internacionales: positivo o negativo. El primero de los casos se trata de la capacidad de algún actor para negociar e influir que otros decidan y se comporten de la manera deseada y el segundo hace referencia a la capacidad de algún actor para evitar que otros ejerzan su influencia sobre las decisiones y comportamiento propios.

Ambos escenarios - positivo y negativo- referentes al uso del poder deben tenerse presentes al tratar de formar coaliciones, no sólo porque la cooperación nunca será perfecta debido a que todas las relaciones internacionales contienen a la vez elementos de conflicto y de cooperación (Del Arenal, 1983); sino porque han propiciado que se suscite una ampliación de alternativas o formas de ejecutar la cooperación multilateral al desarrollo siendo necesario reformar los elementos y enfoques del multilateralismo (Caamaño, 2014). Al respecto, Caamaño (2014) argumenta que los elementos del multilateralismo son complicados y se llevan a cabo en la práctica ya que en relación con el acuerdo señala que podría tratarse de un multilateralismo forzado donde el Estado con mayor poder impone a los demás actores sus ideologías e intereses. En diversas ocasiones no es incluyente ya que se condicionan una serie de principios políticos que se deben cumplir -democracia, respeto a derechos humanos entre otros- para ser miembro del organismo multilateral. Es incipiente la igualdad 
ya que no todos los Estados miembros tienen los mismos derechos y obligaciones más bien se denota que los Estados con mayor poder exigen mayores derechos y privilegios; el control y la supervisión son complicados que se lleven a cabo dado que los Estados pueden ver afectados sus intereses y algunos ámbitos sensibles de su soberanía.

Con base en las contradicciones teórico-prácticas que se presentan en la escena de la cooperación multilateral se puede argumentar que los Ols se enfrentan a lo que Dahl, R. (1994 citado en Innerarity, 2012, p. 14) sintetizaba como "el dilema fundamental de la política en la era de la globalización; la contradicción entre efectividad del sistema y participación ciudadana". Por lo tanto, en virtud de esta situación, se considera que cobra mayor relevancia analizar el papel de los Ols en el diseño de políticas de desarrollo bajo un enfoque teórico-práctico. Por lo que en el siguiente apartado se presentan algunos estudios empíricos que nutren las discusiones sobre ¿Qué comportamiento, estrategias de negociación, discursos e intereses implementan los Ols para incentivar, cabildear y coordinar las negociaciones entre actores estatales (Estado) y no estatales en la escena de la cooperación democrática internacional y formulación de políticas de desarrollo?

\section{Organismos internacionales, actores estatales y no estatales en el diseño de políticas de desarrollo}

En la literatura se pueden encontrar diversos estudios que argumentan que la proliferación de los Ols ayudará a promover el dialogo, resolver los conflictos interestatales que surjan y facilitará la cooperación internacional para solventar los problemas globales ubicados en la agenda internacional del desarrollo mediante el diseño e implementación de políticas que aborden aspectos como: la provisión de bienes públicos globales (PNUD, BM); la paz y la seguridad en el mundo (ONU); el financiamiento a proyectos de desarrollo en los países más pobres (BM); los rezagos sanitarios o problemáticas en el sector salud (OMS); los rezagos educativos (UNESCO); la lucha contra la pobreza (BM, ONU y CEPAL); la promoción del trabajo (OIT); el conocimiento a través de investigaciones científicas (BM, OCDE, FMI) entre muchas otras temáticas más. Sin embargo, particularmente en el área de desarrollo Barkin (2006, p. 103) sostiene que: 
.[... no existe una institución predominantemente equivalente en el área de desarrollo. El papel de las organizaciones internacionales en el desarrollo puede dividirse en tres categorías: préstamos al desarrollo, asistencia para el desarrollo y discursos sobre el desarrollo [...] es necesario destacar que las principales instituciones en cada una de estas tres categorías son: el Banco Mundial (BM), el Programa de las Naciones Unidas para el Desarrollo (PNUD) y la Conferencia de las Naciones Unidas sobre Comercio y Desarrollo (UNCTAD), respectivamente.

En relación con lo señalado por Barkin (2006) se pueden encontrar otros estudios que examinan la evolución del concepto de desarrollo y cómo se ha ido incursionando en los discursos de los Ols, de tal manera que en las décadas de los años cincuenta y sesenta del siglo xx el discurso sobre el desarrollo de los Ols se centró fundamentalmente en las inversiones en capital físico. En la década de los años setenta el interés por el desarrollo se focalizó por el crecimiento económico, mientras que desde finales de los años ochenta y principios de los años noventa el concepto de desarrollo se empezó a considerar como un desarrollo integral y sostenible (Calvo, 2001, pp. 47-48). En la actualidad algunas tendencias del discurso de un OI giran en torno a la generación de alternativas de acción colectiva para superar los problemas globales y alcanzar los objetivos de desarrollo (Barkin, 2006). Con base en estos estudios se reflexiona que los Ols comparten dos características: son grandes burocracias y realizan funciones especializadas en diversas áreas. Sin embargo, es importante resaltar que no existe un OI especialista tanto en el área de desarrollo como en el sector energético. Además, se discute poco sobre ¿por qué los Estados deciden incursionar o salirse de un Ol?, o ¿cómo y bajo qué circunstancias condicionan los Estados otorgar autoridad a un OI?

Al respecto, en Morris y Gleave (2016), Guzman (2013) y Hawkins, et al. (2006) se identificaron algunas situaciones donde un Estado condiciona para participar y delegar autoridad en un OI, tales como: ampliar sus beneficios a través del «free riding ", facilidad para obtener y cubrir financiamientos por parte del FMI y BM, en el caso de los países subdesarrollados tener la oportunidad de transformarse en un país desarrollado, ventaja tecnológica, uso de recursos naturales, liderar una agenda de desarrollo multilateral, administrar externalidades de políticas, resolver disputas, aumentar la credibilidad democratizadora, entre otras. Mientras tanto en Caraway, et al. (2012); Mansfield y Pevehouse (2006) y Tago (2005) se pueden encontrar que algunas condiciones internas de un país como la recesión, el ciclo electoral, los intereses de los ciudadanos, el gobierno dividido y la democratización crean diferentes incentivos 
para que el Estado busque compartir la carga con sus aliados o busque legitimidad colectiva en los Ols. En particular Mansfield y Pevehouse (2006) matizan que los Estados que están pasando por transiciones democráticas tienen fuertes incentivos para unirse a los OI, ya que hacerlo proyecta una imagen legitima al resto del mundo al mostrar que los esfuerzos de reforma y de participación política son sinceros.

Con base en lo anterior y considerando a Tilly (2010), quien aporta principios para la descripción y comprensión de la democracia, la democratización y la desdemocratización, es posible señalar que existe una correlación positiva entre democratización y los Ols. Se discurre que la democratización es un proceso que tiene como producto fortalecer las relaciones entre el Estado y la sociedad civil en el proceso de toma de decisiones, por lo que es posible rechazar la idea de que solamente los actores políticos del Estado negocian para establecer la agenda y diseñar políticas públicas, sino que la sociedad civiljuega un papel importante en la mesa de negociación doméstica ya que puede exigir, mediante elecciones competitivas, presión política, encuestas de opinión y movimientos sociales participar en los procesos de toma de decisiones y exigir una mejor representatividad política tanto en el contexto nacional como internacional.

Este último aspecto se ve materializado con la incursión o salida del Estado en Ols, tal es el caso del Brexit. Al respecto, Mangas (2016) y Booth (2016) argumentan que las principales razones por las que el Reino Unido abandonó la Unión Europea son: recuperar soberanía, divergencias respecto a la política de refugiados y migración y porque no se redujeron los poderes de la Unión Europea. A pesar de estas razones es importante señalar que el resultado del Brexit no fue uniforme en todo el Reino Unido, el 52 \% de la sociedad civil votó a favor de la salida en Inglaterra y Gales, mientras que el $48 \%$ en Escocia, Irlanda del Norte y Gibraltar votaron por la permanencia. Es claro que existe una fuerte divergencia de intereses en la sociedad civil, ¿a qué se debe esto?

Se considera que un factor determinante y que no ha sido abordado de manera amplia y profunda, en temas de desarrollo, es la cultura. Según Radcliffe (2006) la cultura comprende los patrones de las relaciones sociales y las estructuras de los sentimientos producidos por múltiples actores, que están diferencialmente posicionados en las relaciones de poder, las economías políticas y la reproducción social. En el caso del Brexit, se discurre que elementos como la educación y la política cultural, así como las ideologías y patrones de pertenencia por parte de la sociedad civil jugaron un papel fundamental en el proceso de toma de decisiones, destacando el fuerte conflicto de intereses que fracturó la cohesión social. Por lo tanto, es posible señalar que la capacidad y poder de negociación del Estado, la política interna, la cohesión 
social y las formas no estatales de poder definen las posibilidades de democratización, incursión o salida del Estado de un Ol. Esta idea es expuesta con mayor claridad por Mansfield y Pevehouse (2006, p. 163) quienes señalan que:

[...] un ímpetu clave del Estado a la membresía de un Ol emana de la arena política doméstica [...]. Aunque los estudios de las instituciones internacionales han puesto relativamente poco énfasis en los efectos de la política interna, los resultados de nuestro análisis sugieren fuertemente que la reciente ola de democratización probablemente expandirá el tamaño y el número de Ol. Además, es probable que esta expansión sea un buen augurio para la supervivencia a largo plazo de la democracia.

Dado el fuerte vínculo entre la democratización y un OI (ver caso OCDE) se considera relevante analizar las condiciones en las que los Ols promueven, o no, la democratización (Pevehouse, 2005) así como el papel que desempeñan en la escena de la cooperación democrática internacional y formulación de políticas de desarrollo con actores estatales y no estatales. Para llevar a cabo este último análisis se consideran dos mesas de negociación: internacional y doméstica. Esto bajo la justificación que la política interna puede tener un efecto directo en la política internacional y viceversa. Para ilustrar mejor las limitaciones y metas de los formuladores de políticas tanto a nivel nacional como internacional se retoma a Putnam (1988, p. 434) quien desarrolla el enfoque del juego de dos niveles, en el que señala que las interacciones domésticas e internacionales son mutuamente dependientes afirmando que las preferencias de los actores domésticos definen los términos de la cooperación internacional dado que:

[...] a nivel nacional los grupos nacionales persiguen sus intereses presionando al gobierno para que adopte políticas favorables y los políticos busquen el poder al construir coaliciones entre esos grupos. A nivel internacional, los gobiernos nacionales tratan de maximizar su propia capacidad de satisfacer las presiones internas, al tiempo que minimizan las consecuencias adversas de los desarrollos extranjeros [...] cada líder político nacional aparece en ambos tableros del juego. A través de la mesa internacional se sientan sus homólogos extranjeros, y en sus codos se sientan diplomáticos y otros asesores internacionales. Alrededor de la mesa doméstica se encuentran figuras de partidos y parlamentarios, portavoces de agencias nacionales, representantes de grupos de interés clave y asesores políticos del líder. 
Cabe destacar que dichas mesas de negociación se caracterizan por contar con un actor internacional y múltiples actores estatales y no estatales, así como múltiples asuntos que requieren reglas bien definidas, elecciones e información para maximizar sus beneficios y expectativas. Dado el interés de este trabajo de indagación, a continuación se presentan algunos casos que denotan los intereses y las negociaciones entre los Ols y los actores estatales -Estado-nación- y no estatales -empresas transnacionales y sociedad civil- en la escena de la cooperación democrática internacional y formulación de políticas de desarrollo (ver figura 2).

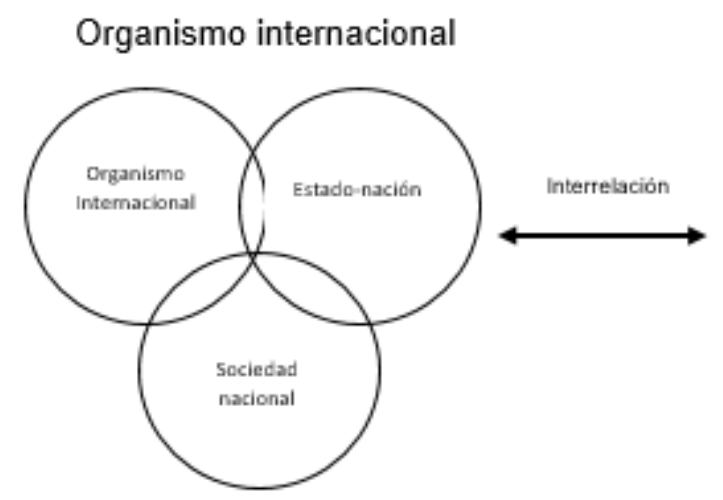

\section{Negociación doméstica}

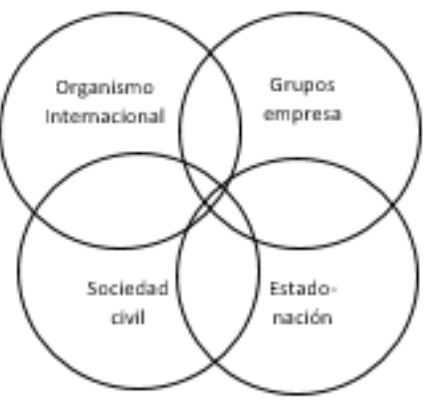

Figura 2. Mesas de negociación: internacional y doméstica.

Fuente: elaboración propia

\section{Actores estatales. Estado-nación}

Los Ols influyen en las políticas de desarrollo y promueven la cooperación multilateral de varias maneras tales como, generando información, empoderando a los expertos en la formulación de política interna, persuadiendo a los gobiernos miembros a tomar medidas que sean de su propio interés y mediante elecciones democráticas y agendas privadas (Fang, 2008; Krause, 2014). Particularmente Fang y Stone (2012) mediante un modelo de teoría de juego considerando el enfoque del agente -el experto en política interna- y el principal -el Ol- sostienen que la eficacia de las instituciones internacionales como asesores de políticas depende de la polarización de la política interna, persuasión y la confianza. Destacando que "la persuasión sólo es posible si el gobierno confía en el Ol como fuente de información y la confianza sólo es posible si la verdad es una estrategia óptima para los Ols" (Fang y Stone, 2012, p. 7).

La confianza es un factor clave para que los Ols cumplan con sus funciones. Sin embargo, se considera que es inherentemente problemática, porque existe un dilema respecto a la representatividad de un OI ya que dichos agentes internacionales 
agregan los intereses de sus miembros, que es poco probable que estén alineados con los expertos de la política interna de un Estado en particular. En este sentido, con base en Fang y Stone (2012) se discurre que los expertos en política interna juegan un doble papel en cuanto al alcance y persuasión de los Ols para formar coaliciones e influir en el diseño de políticas de desarrollo. Por un lado, los OI son más influyentes cuando el gobierno nacional no confía en los expertos en política interna; y por otro lado, cuando los gobiernos nacionales confían en sus expertos de política interna, desconfían "del asesoramiento de los organismos internacionales, porque saben que sus preferencias divergen" (Fang y Stone 2012, p. 25). Algunos acontecimientos o evidencia empírica que podría sustentar la creciente desconfianza que se ha presentado en los últimos años por parte de los expertos de política interna hacia los Ols se observan en la tabla 3. Destacando que dicha evidencia gira en torno a examinar ¿a qué o a quién (es) representa un Ol?

Tabla 3. Evidencia empírica que refleja desconfianza en los Ol: ¿a qué o a quién (es) representa un organismo internacional?

\begin{tabular}{|c|c|c|}
\hline $\begin{array}{c}\text { Organismo } \\
\text { internacional }\end{array}$ & Acontecimientos & Fuente consultada \\
\hline ONU & $\begin{array}{l}\text { El poder de veto del Consejo de Seguridad de las Naciones } \\
\text { Unidas está conformado por cinco miembros permanentes } \\
\text { que son China, Francia, Rusia, Reino Unido y Estados Unidos. } \\
\text { Esto aumenta el poder de negociación de los agentes } \\
\text { estadounidenses franceses y demás miembros, pero también } \\
\text { reduce el alcance de la cooperación internacional ya que } \\
\text { existen situaciones en las que "los } 5 \text { grandes" buscan la } \\
\text { protección de sus propios intereses, por lo que aumentan las } \\
\text { probabilidades de defección involuntaria -de otros países- de } \\
\text { la organización mundial en aras del unilateralismo. }\end{array}$ & Torres (2008) \\
\hline FMI & $\begin{array}{l}\text { Aliados más cercanos de los Estados Unidos (y otros países } \\
\text { del Grupo de los 7) recibieron préstamos del FMI con menos } \\
\text { condiciones, especialmente antes de las elecciones. }\end{array}$ & Broz y Brewster (2006) \\
\hline \multirow[b]{2}{*}{ FMl y BM } & $\begin{array}{l}\text { El director del FMI es siempre un europeo y el presidente del } \\
\text { Banco Mundial es siempre un estadounidense. El Secretario } \\
\text { General de las Naciones Unidas es seleccionado de una lista } \\
\text { de los candidatos regionales en forma rotativa. }\end{array}$ & Hawkins, et al. (2006) \\
\hline & $\begin{array}{l}\text { El Banco Mundial y el Fondo Monetario Internacional } \\
\text { imponen a los Estados en vías de desarrollo, a través del } \\
\text { denominado "Consenso de Washington", políticas de } \\
\text { liberalización, de privatización, de ajuste estructural y de } \\
\text { desregulación, como parte de la renegociación de la deuda } \\
\text { externa que asfixiaba a dichos países. }\end{array}$ & Del Arenal, (2008) \\
\hline
\end{tabular}

Fuente: elaboración propia con base en los autores citados en la tabla 
En relación con los acontecimientos mostrados en la tabla 3, es evidente que existe una cúpula o élite de poder internacional que se resisten a cualquier reducción de poder, por lo que merman la percepción de confianza, alcances y autonomía de los Ols. Es común que dicha cúpula de poder sólo busque la protección de sus propios intereses y trate de influir en el comportamiento y decisiones del Ol, principalmente, a través de sus derechos de voto, el nombramiento de personal y la retirada de las contribuciones financieras. Este conflicto de intereses refleja, en buena medida, las limitaciones de los Ols para llegar a un acuerdo, en diversas ocasiones no tienen ni medios de fuerza independientes ni el derecho a regular los actores estatales y no estatales con autoridad.

Aunado a lo anterior, otro factor que limita las funciones de los Ols es el poder de los líderes o funcionarios de los Ol ya que pueden influir y dar forma al comportamiento tanto de los Ols como de los Estados miembros. Tal es el caso del presidente del Banco Mundial quien puede influir en el comportamiento de los Estados, ya que dicho líder con base en sus intereses e ideologías puede buscar persuadir que el modelo neoliberal se imponga como óptimo para que los países subdesarrollados crezcan y se desarrollen económicamente a través de la liberalización del mercado. De esta manera, con base en lo anterior, se considera que el papel de los Ols en el diseño de políticas de desarrollo puede ser analizado desde dos perspectivas de acuerdo con la representatividad de intereses, nivel de autonomía y su capacidad de negociación: 1) los Ols con capacidad flexible de negociación y poca autonomía que son aquellos codependientes de los intereses de los Estados que los conforman; y 2) los Ols con capacidad autoritaria que representan sus propios intereses como agente autónomo.

En el primero de los casos los intereses y las preferencias de los Estados miembros representan una limitante a la autonomía y capacidad del OI para cumplir con sus funciones. Tal es la situación de la Unión Europea que sus capacidades de formulación de políticas son estrictamente limitadas, porque dependen de las negociaciones multilaterales entre los gobiernos nacionales, las cuales según Scharpf (1994) son engorrosas y son fácilmente bloqueadas por los conflictos de intereses entre los Estados miembros. Mientras que en el segundo de los casos, Guzman (2013) señala que si el comportamiento de los Ols es autoritario los Estados no tendrían autonomía ni capacidad de decisión mediante sus propias políticas y se estarían enfrentando al «problema de Frankenstein" ya que un Ol puede convertirse en un monstruo que los puede perjudicar, en lugar de ayudar.

La implicación de ambos casos conlleva a una versión global del dilema del prisionero: "ningún país puede tener éxito por sí solo, pero ninguno confía lo suficiente 
en los otros para procurar una cooperación y coordinación eficaces" (Ocampo, 2015, p. 17). De esta manera, ante la falta de confianza y la evidente divergencia de intereses entre Estados y Ols, emerge la pregunta: ¿en qué condiciones puede un Ol obtener credibilidad de información y confianza por parte de un gobierno que no comparte sus preferencias? Algunas de las estrategias y discursos legitimadores que los Ols han considerado implementar en los últimos años para alcanzar la conciliación de intereses en el plano internacional y la cooptación en los sistemas políticos nacionales son: ofrecer incentivos para mejorar el diseño de políticas internas en los Estados miembros y cambiar tanto el discurso ideológico de desarrollo por desarrollo sostenible, como el de buen gobierno por la aplicación del concepto de buena gobernanza (Enríquez, 2015; Calvo, 2001). Al respecto es posible destacar el caso de la OMS, que ha persuadido para incluir los temas de salud mental en las agendas de salud doméstica de países donde estas cuestiones no se hubieran discutido dadas sus ideologías, como es el caso de China (Meng, et al. 2004). Al respecto, Huang (2015) destaca cómo las políticas de salud doméstica pueden ser moldeadas por la OMS, incluso en el caso de Estados poderosos como China, que generalmente son muy protectores de su soberanía. Según Huang (2015), entre los mecanismos y estrategias que implementó la OMS para persuadir a los dirigentes chinos destacan: la financiación de proyectos de salud condicionada, ayudando a identificar nuevos problemas de salud, promoviendo innovaciones de políticas y facilitando el aprendizaje y la internalización de las normas. Los resultados de dicha investigación confirman que:

La cooperación efectiva es más probable cuando las externalidades negativas del comportamiento del Estado de China son sustanciales y las reglas de salud, estrategias o mecanismos son diseñados apropiadamente. La cooperación es mucho más complicada cuando el problema de salud global en cuestión no tiene significancia externa. (Huang, 2015, p. 66).

El caso de China puede representar una ventana de oportunidad para que Estados autoritarios no tomen posturas proteccionistas sobre el tema de la cooperación al desarrollo. Se plantea entonces: ¿es posible que los Ols logren persuadir a los actores estatales y no estales para formar coaliciones en el diseño de políticas de desarrollo? 


\section{Actores no estatales. Empresas transnacionales}

Los principales objetivos de cualquier empresa transnacional son maximizar sus beneficios y reducir sus costos, sin embargo, se considera que existen situaciones en las que los cambios en las normas de salud y seguridad, normas de protección ambiental, regulación de los medios de comunicación, incrementos de aranceles o la política económica general de los gobiernos extranjeros afectan tanto su capacidad para comerciar como sus ganancias.

Ante esta situación, Allard (2007) señala que las empresas transnacionales no aceptaran perder posicionamiento del mercado ni recursos financieros por lo que pueden cambiar su rol económico empresarial y transformarse en actores en el plano de la política internacional. En este sentido, Willetts (2001) argumenta que las empresas son un grupo de poder que ante la posibilidad de perder privilegios pueden decidir ejercer presión política sobre el gobierno extranjero siguiendo alguna de las siguientes cuatro rutas: 1) indirectamente por la empresa pidiendo a su propio gobierno presionar al gobierno extranjero; 2) indirectamente planteando una cuestión de política general en una organización internacional; 3) directamente en el país a través de la embajada diplomática; o 4) directamente en el otro país a través de los ministerios gubernamentales.

Con base en los puntos señalados por Willetts (2001) se discurre que las empresas juegan un papel importante tanto en la política nacional como en la internacional. En un primer momento la élite empresarial puede participar en asociaciones empresariales, contribuir con financiamientos a campañas electorales de ciertos partidos políticos y participar en prácticas corruptas con la finalidad de ejercer presión política al Estado para que éste incursione en un Ol. En un segundo momento, es oportuno señalar que las empresas transnacionales también pueden jugar un papel importante en el diseño de políticas de desarrollo. Tal es el caso de un consenso entre el Estado, las universidades y la sociedad civil para que se vinculen y generen conocimiento e investigaciones en pro del desarrollo, o que estas puedan ser un sustento considerable en la empleabilidad para los actores locales.

\section{Sociedad civil global}

Las redes transnacionales de las organizaciones de la sociedad civil, especialmente las ONG, como la Fundación Bill y Melinda Gates o Greenpeace se han extendido por las fronteras nacionales. Según Milner, et al. (2016) este aumento de organizaciones de la sociedad civil global se debe a que los ciudadanos están más dispuestos a 
mostrar apoyo conductual en favor de proyectos de ayuda externa en comparación con los programas gubernamentales, esto debido principalmente a la incapacidad del gobierno local de entablar un dialogo participativo con los ciudadanos quienes juzgan a su gobierno como corrupto o clientelista.

Ante esta situación, Kumar (2012) sostiene que la sociedad civil global a través de movilizaciones en todo el mundo ha intentado adquirir una mayor voz en los procesos de toma de decisiones de los Ols con la principal intención de proporcionar estrategias de solución más efectivas a los problemas locales que las que podrían establecer los Estados-nación. La sociedad civil global se ha convertido en un factor determinante de las relaciones internacionales contemporáneas (Kumar, 2012). Tal es el caso de la coalición entre el FMI, BM y 26 ONG de todas las regiones geográficas del mundo para formular políticas que vayan encaminadas a revolver problemas de falta de financiamiento al desarrollo, el medio ambiente, la pobreza y el desarrollo participativo en el mundo (Serrano, 1999). Otro caso por destacar es el de Caraway, et al. (2012) quienes examinan mediante estudios de caso -en Grecia, Corea del Sur y Bolivia- y un modelo econométrico si los intereses económicos de los ciudadanos influyen en la condicionalidad de los préstamos laborales del FMI. Los resultados de dicho análisis demuestran que los países democráticos con mano de obra interna más fuerte reciben condiciones laborales menos intrusivas en sus programas de préstamos del FMI. Por lo tanto, el FMI responde a la política interna en los países prestatarios y, en particular, a los intereses de los trabajadores.

Sin duda, estos casos son claros ejemplos que la sociedad civil global desempeña un papel fundamental en el diseño de políticas públicas.

\section{Conclusiones}

El marco teórico que ha guiado este trabajo de investigación hace hincapié en la necesidad de desarrollar una teoría de los organismos internacionales que tome en cuenta el análisis de su comportamiento y funciones. Sumado al papel que desempeñan en la formulación de políticas de desarrollo como agentes estratégicos, negociadores y coordinadores en la cooperación multilateral al desarrollo. Igualmente, sus relaciones tanto con el entorno como con actores estatales y no estatales para formar coaliciones en el diseño de políticas de desarrollo considerando la estructura de poder y la confianza.

Además, con base en los fundamentos teóricos y evidencia empírica presentada en este artículo se discurre que una estrategia eficaz para resolver los problemas 
globales y mejorar las condiciones de vida de la sociedad internacional requiere una red de gobernanza multinivel coordinada por un organismo internacional. Esto bajo la justificación que los organismos internacionales, dado su estructura, objetivos y funciones, cumplen con la mínima institucionalidad que regula el sistema mundial, es una de las entidades que mayor poder político-institucional, científico-técnico, epistemológico y financiero tienen. También pueden integrar, cabildear y coordinar acciones colectivas entre Estado, empresas transnacionales, sociedad civil y otros actores que mejoren las condiciones socioeconómicas, políticas y ambientales, entre y al interior de los países, especialmente de los más pobres a través de la cooperación multilateral al desarrollo.

Se observó que para que un organismo internacional cumpla con sus funciones y expectativas requiere fortalecer la democratización y contar con buena percepción de confianza por parte de los actores estatales y no estatales. Con la revisión de la literatura y desde una perspectiva generalizable a continuación se presentan algunas estrategias que podrían ser implementadas por cualquier Ol -independientemente de su especialización- para mejorar tanto la confianza en la cooperación interestatal como la formulación de políticas de desarrollo. Algunas son: construir una red fuerte de instituciones regionales para salvaguardar el orden internacional (Ocampo, 2013); promocionar la transparencia institucional, la rendición de cuentas y el seguimiento posapoyo al desarrollo con la finalidad de superar los problemas asociados con la asimetría de información evitando holguras o comportamientos no deseados (Hawkins, et al. 2006); recompensar a los agentes por acciones deseadas o buenas prácticas, a través de mayores montos de financiamiento y expansiones presupuestarias (Barkin, 2006); sucitar elecciones competitivas en el nombramiento de los dirigentes de los Ols donde "las elecciones nacionales pueden contribuir de algún modo a disminuir el déficit democrático endémico de las instituciones internacionales" (Rickard y Caraway, 2014:716); diseñar normas específicas para tratar la discusión sobre le legalidad de actos ultra vires, mecanismos de impugnación y controles de legalidad de los actos de los Ols (Querejazu, 2013); asegurar la participación equitativa de los países en vías de desarrollo en la gobernanza mundial (Ocampo, 2013); incrementar la integración de las redes de confianza dentro de la política pública; y disminuir la autonomía de los principales centros de poder de la política pública (Tilly, 2010).

Se considera que si este conjunto de estrategias funcionan puede proporcionar un mecanismo por el cual los Ols pueden ejercer una influencia socializadora basada en la confianza y trabajo multinivel donde los expertos o tomadores de decisiones internos coordinarán, gradualmente, las políticas de desarrollo para alinearlas con las normas y objetivos internacionales del desarrollo y así trabajar colaborativamente entre 
actores estatales y no estatales en busca de generar estrategias de desarrollo para mejorar las condiciones de vida de la sociedad a nivel internacional, nacional y local.

Aunque se considera que las acciones anteriores son complicadas de efectuar cabalmente por el conflicto de intereses que existen, o podrían existir, en las relaciones internacionales entre actores estatales y no estatales no es imposible de que se lleven a cabo. Como se pudo observar en este trabajo existen casos especiales como el de OMS-China que son consideradas como ventanas de oportunidad para que Estados autoritarios no tomen posturas proteccionistas sobre el tema de la cooperación al desarrollo y se puedan replicar en otros países y en otras áreas de desarrollo.

\section{Referencias}

Albi, E. (2005). La globalización económica como marco de las relaciones internacionales. ICE, 825, 9-18. Recuperado de: https://dialnet.unirioja.es/servlet/articulo?codigo=1709410

Allard, R. (2007). Las empresas multinacionales en la globalización. Relaciones con los Estados. Estudios Internacionales, 158, 59-99. Recuperado de: https://revistaei.uchile.cl/index.php/ REl/article/view/14154

Álvarez, S. (2012). Una introducción a la cooperación internacional al desarrollo, REDUR, 10, 285309. Recuperado de: file:///C:/Users/p.indesco.nal/Downloads/Dialnet-UnalntroduccionAL aCooperacionInternacionalAIDesarr-4104832.pdf

Babb, S.y Chorev, N. (2016). International organizations: looseand tightcoupling in the development regime. Studies in Comparative International Development, 51(1), 81-102. Recuperado de: https://www.springerprofessional.de/en/international-organizations-loose-and-tightcoupling-in-the-deve/10036866

Baldwin, D. (1993). Neoliberalism, neorealism and world politics. In D. Baldwin. (Ed.), Neorealism and Neoliberalism. The Contemporary Debate. Nueva York, USA: Columbia University Press. Recuperado de: file:///C:/Users/p.indesco.nal/Downloads/Baldwin\%20(1993).pdf

Bárcena, A. (2015). La descolonización de la agenda para el desarrollo después de 2015: hacia una asociación universal para el desarrollo. En J. A. Ocampo. (Ed.), Gobernanza global y desarrollo: Nuevos desafíos y prioridades de la cooperación internacional, Buenos Aires, Argentina: Siglo Veintiuno Editores. 
Barkin, J. S. (2006). International organization: theories and institutions. USA: Palgrave Macmillan. Recuperado de: https://stackofideas.files.wordpress.com/2012/02/j-_samuel_barkin_international_organization_thbookfi-org.pdf

Booth, S. (2016). El Brexit: un desafío para todo el continente. Fundación Faes, 185, 1-14. Recuperado de: https://fundacionfaes.org/file_upload/publication/pdf/20160310180423el _-brexit__un_desafio_para_todo_el_continente.pdf

Broz, J. L. and Brewster, M. (2006). US domestic politics and International Monetary Fund policy. In D. G. Hawkins, et al. (Ed.), Delegation and Agency in International Organizations, New York, USA: Cambridge University Press.

Caamaño, L. (2014). La eficacia del multilateralismo en las relaciones internacionales. Revista del Instituto Español de Estudios Estrategicos, 1, 1-16. Recuperado de: http://www.ieee. es/Galerias/fichero/docs_opinion/2014/DIEEEO87-2014_EficaciaMultilateralismo_Luis Caamano.pdf

Calvo, A. (2001). Los organismos internacionales y la cooperación al desarrollo. Revista de Economía Mundial, 5, 47-61. Recuperado de: https://www.sem-wes.org/sites/default/files/revistas/rem5 -3.pdf

Caraway, T. L., et al. (2012). International negotiations and domestic politics: the case of IMF labor market conditionality. International Organization, 66, 27-61. Recuperado de: http:// personal.Ise.ac.uk/RICKARD/imf.pdf

Dahl, R. (1957). The concept of power. Behavioral Science, 2(3), 201-215. Recuperado de: https://welcometorel.files.wordpress.com/2008/08/conceptpower_r-dahl.pdf

Del Arenal, C. (1983). Poder y relaciones internacionales: un análisis conceptual contundentes. Revista de Estudios Internacionales, 3(3), 501- 524. Recuperado de: file://C:/Users/ p.indesco.nal/Downloads/Dialnet-PoderYRelaciones/nternacionales-2496100.pdf

Del Arenal, C. (2008). Mundialización, creciente interdependencia y globalización en las relaciones internacionales. Cursos de derecho internacional y relaciones internacionales de VitoriaGasteiz. España: Universidad del País Vasco. Recuperado de: https://www.ehu.eus/es/ web/cursosderechointernacionalvitoria/-/mundializacion-creciente-interdependencia-yglobalizacion-en-las-relaciones-internacionales 
Enríquez, I. (2015). Los organismos internacionales y su incidencia en el desarrollo latinoamericano: la gravitación del pensamiento y las estrategias del Banco Mundial en las políticas públicas mexicanas. España: Universidad Complutense de Madrid. Recuperado de: https://eprints. ucm.es/33678/

Fang, S. and Stone, R. (2012). International organizations as policy advisors. International Organization, 66(4), 537-569. Recuperado de: https://www.cambridge.org/core/journals/ international-organization/article/international-organizations-as-policy-advisors/55E7191 2941F8A2F69BDB92CFC47A221

Fang, S. (2008). The Informational role of international and domestic politics. American Journal of Political Science, 2(2), 304-321. Recuperado de: https://onlinelibrary.wiley.com/doi/ abs/10.1111/j.1540-5907.2007.00314.x

Fernández, N. y Schejtman, L. (2012). Planificación de políticas, programas y proyectos sociales. Buenos Aires, Argentina: Fundación CIPPEC. Recuperado de: https://www.cippec.org/wpcontent/uploads/2017/03/1419.pdf

Giraldo, G. (2015). El origen de la gobernanza global y su propósito: la justicia social. España: Universidad de Valencia. Recuperado de: https://www.educacion.gob.es/teseo/ imprimirFicheroTesis.do?idFichero=hf5gGot29Wc\%3D

Guy, P. B. (2007). Globalización, gobernanza y Estado: algunas proposiciones acerca del proceso de gobernar. Revista del CLAD Reforma y Democracia, 39, 33-50. Recuperado de: http://old.clad.org/portal/publicaciones-del-clad/revista-clad-reforma-democracia/ articulos/039-octubre-2007/0055723-1

Guzman, A. (2013). International organizations and the frankenstein problem. The European Journal of International Law, 24(4), 999-1025. Recuperado de: https://academic.oup.com/ ejil/article/24/4/999/606374

Hawkins, D. G., et al (2006), Delegation and Agency in International Organizations. New York, USA: Cambridge University Press. Recuperado de: https://www.cambridge.org/ core/books/delegation-and-agency-in-international-organizations/B28F1B57D432A034A 83938C776DE1A85

Huang, Y. (2015). International institutions and China's health policy. Journal of Health Politics, Policy and Law, 40(1), 41-71. Recuperado de: file:///C:/Users/p.indesco.nal/ Downloads/Huang-International_institutions_and_Chinas_health_policy.pdf 
Innerarity, D. (2012). La gobernanza global, de la soberanía a la responsabilidad. Revista CIDOB d'Afers Internacionals, 100, 11-23. Recuperado de: https://www.cidob.org/es/ articulos/revista_cidob_d_afers_internacionals/100/la_gobernanza_global_de_ la_soberania_a_la_responsabilidad

Keohane, R. O. and Nye, J. S. (1977). Power and interdependence: world politics in transition. Boston, USA: Little, Brown. Recuperado de: https://www.worldcat.org/title/power-andinterdependence-world-politics-in-transition/oclc/2748258

Karns, M., et al. (2015). International organizations: the politics and processes of global governance. USA: Lynne Rienner Publishers. Recuperado de: https://www.rienner.com/uploads/55b14 c992d1b2.pdf

Krause, M. (2014). Organismos internacionales y transformaciones del Estado en salud y educación. Estudios, 31, 115-134. Recuperado de: file:///C:/Users/p.indesco.nal/Downloads/Dialnet -OrganismosInternacionalesYTransformacionesDelEstad-5209713.pdf

Kumar, V. (2012). The role of global civil society in global governance. Beijing Law Review, 3, 206212. http://m.scirp.org/papers/25725

Mangas, A. (2016). Los dilemas del Reino Unido y de la UE: ¿salir o cambiar la Unión? Real Instituto Elcano. Recuperado de: http://www.realinstitutoelcano.org/wps/portal/rielcano _es/contenido?WCM_GLOBAL_CONTEXT=/elcano/elcano_es/zonas_es/europa/dt3-2016 -mangasmartin-dilemas-reino-unido-ue-salir-cambiar-union

Mansfield, E. D., y Pevehouse, J. C. (2006). Democratization and international organizations. International Organization, 60, 137-167. Recuperado de: https://www.cambridge.org/core/ journals/international-organization/article/democratization-and-international-organizatio ns/8DC78F62EC16B09B5C254A6CBCBCAC03

Meadows, D. H., et al. (1972). The limits to growth. A report for the club of Rome's project on predicament of mankind. New York: Universe Books. Recuperado de: https://archive.org/ details/TheLimitsToGrowth/page/n9

Meng, Q., et al. (2004). Health policy and systems research in China. Special programme for research and training in Tropical Diseases (TDR). Recuperado de: https://www.who.int/tdr/ publications/documents/health-research-china.pdf 
Milner, H., et al. (2016). Citizen preferences and public goods: comparing preferences for foreign aid and government programs in Uganda. The Review of International Organizations, 11(4), 219-245. Recuperado de: https://link.springer.com/article/10.1007/s11558-016-9243-2

Morris S. and Gleave, M. (2016). Realizing the power of multilateralism in US development policy. Center for global development, 1-10.

Ocampo, J. A. (2013). Repensar la economía global y la gobernanza social. En J. E. Stiglitz y M. Kaldor. (Ed.), La búsqueda de la seguridad, protección sin proteccionismo y el desafío de la gobernanza global. México: Paidos.

Ocampo, J. A. (2015). La gobernanza económica y social y el sistema de las Naciones Unidas. En J. A. Ocampo. (Ed.), Gobernanza global y desarrollo: Nuevos desafíos y prioridades de la cooperación internacional. Buenos Aires, Argentina: Siglo Veintiuno Editores. Recuperado de: https://repositorio.cepal.org/handle/11362/38855

Pevehouse, J. C. (2005). Democracy from above regional organizations and democratization. USA: Cambridge University Press. Recuperado de: https://www.amazon.com/Democracy -Above-Regional-Organizations-Democratization/dp/0521844827

Putnam, R. D. (1988). Diplomacy and domestic politics: the logic of two-level games, International Organization,3(42),427-460. Recuperado de: http://www.guillaumenicaise.com/wp-content /uploads/2013/10/Putnam-The-Logic-of-Two-Level-Games.pdf

Querejazu, A. (2013). Responsabilidad de las organizaciones internacionales por actos Ultra vires. Revista de derecho, 39, 1-29 Recuperado de: http://www.scielo.org.co/scielo. php?pid=S0121-86972013000100001\&script=sci_abstract\&tlng=es.

Radcliffe, S. A. (2006). Culture in development thinking: geographies, actors and paradigms. En A. R. Sarah. (Ed.). Culture and development in a globalizing world, geographies, actors and paradigms. Nueva York, USA: Routledge.

Restrepo, J. C. (2013). La globalización en las relaciones internacionales: Actores internacionales y sistema internacional contemporáneo. Revista Facultad de derecho y ciencias políticas, 43(119), 625-654. Recuperado de: https://revistas.upb.edu.co/index.php/derecho/ article/view/2360 
Rickard, S. J. and Caraway, T. L. (2014). International negotiations in the shadow of national elections. International Organization, 68(3), 701-720. Recuperado de: https://www.cambridge.org/ core/journals/international-organization/article/international-negotiations-in-the-shadow-of-national-elections/7895DC7630330C40747F9F6C40EA696E

Robinson, D. J. (2014). Economic development from the state and local perspective: case studies and public policy debates. USA: Palgrave Macmillan. Recuperado de: https://www.amazon.com/ Economic-Development-State-Local-Perspective-ebook/dp/B00PITDBEU

Salomón, M. (2002). La teoría de las Relaciones Internacionales en los albores del siglo XXI. Revista CIDOB d'Afers Internacionals, 56, 7-52 Recuperado de: https:/www.raco.cat/index.php/ revistacidob/article/viewFile/28242/28076

Sanahuja, J. A. (2008). ¿Un mundo unipolar, multipolar o apolar? El poder estructural y las transformaciones de la sociedad internacional contemporánea, en Gasteiz, V. Cursos de Derecho Inter nacional. Bilbao: Servicio Editorial de la Universidad del País Vasco. Recuperado de: https://eprints.ucm.es/42540/1/Un_mundo_unipolar_multipolar_o_apolar_E. pdf

Serrano, E. (1999).Las ONG comoactores delas relaciones internacionales. Universidad Complutense de Madrid: España. Recuperado de: https://eprints.ucm.es/2019/1/T23903.pdf

Scharpf, F. W (1994). Community and autonomy: multi-level policy-making in the European Union. Journal of European Public Policy, 1(2), 219-242. Recuperado de: https://www.tandfonline. com/doi/abs/10.1080/13501769408406956

Stiglitz, J. E. (2006). Making globalization work. New York, USA: Norton Recuperado de: https:// www.fundacionsistema.com/wp-content/uploads/2015/05/PPios7_Making-GlobalizationLIBRO.pdf

Stiglitz, J. E. (2013). Protección Social sin proteccionismo. En E. Stiglitz y M. Kaldor. (Ed.), La búsqueda de la seguridad, protección sin proteccionismo y el desafío de la gobernanza global. México: Paidos. https://www. planetadelibros.com.ar/libro-la-busqueda -de-la-seguridad/132335

Tago, A. (2005). Determinants of multilateralism in us use of force: states of economy, election cycle, and divided government. Journal of Peace Research, 5(42), 585-604. Recuperado de: https://journals.sagepub.com/doi/abs/10.1177/0022343305056235 
Tilly, C. (2010). Democracia. Madrid:Akal. Recuperado de: https://www.akal.com/libro/democracia -34284/

Torres, M. (2008). El derecho de veto en el Consejo de Seguridad de Naciones Unidas: la historia de la válvula de seguridad que paralizó el sistema. ACDI, 1, 49-88. Recuperado de: file:///C:/ Users/p.indesco.nal/Downloads/125-373-1-PB.pdf

Willetts, P. (2001). Transnational actors and international organizations in global politics. In J. B. Baylis and S. Smith, S. (Ed.), The Globalisation of world politics, Oxford and New York, USA: Oxford. Recuperado de: http://biblioteca.cejamericas.org/bitstream/handle/2015/3651/ TransnationalActors.pdf 